\title{
Low Order Fabry-Perot Low Dispersion Multicolor Large Field Survey System
}

\author{
Jiang Shi-Yang \\ Beijing Astronomical Observatory, Chinese Academy of Sciences \\ Beijing 100080, China
}

\begin{abstract}
Crossing a low interference order Fabry-Perot etalon with an objective prism on a Schmidt telescope leads to a simple large field multiband spectrophotometric survey system well-adapted to the study of the spectral energy distribution of faint galaxies and redshift estimates.
\end{abstract}

\section{Introduction}

Objective prism is a kind of low resolution 3-D spectrograph. But its limiting magnitude is set by the sky background. This largely extended source produces at each point on the focal plane a white light background. Recently people found that using several narrow band filters, photometry gives an already sufficient information to recover the low resolution continuum spectra of faint extragalactic objects. An estimate of the redshifts with $1 \%$ accuracy may be derived. However, it is too time consuming to get 10 times $20 \mathrm{~nm}$ band observations till $\mathrm{V}=21.0$. Here we suggest a way to solve this problem to some extent.

As we know a Fabry-Perot(FP) has many orders so that we can have many narrow passbands overlapping each other. If we add an objective prism as cross disperser we can get many narrow band images of the same object on the focal plane. The sky background will be limited by the FP passbands as when we do filter photometry, but we get all colors simultaneously so that we can save a lot of time doing the same thing with much better homogeneity. For passbands of $5 \mathrm{~nm}$ to $20 \mathrm{~nm}, \mathrm{FP}$ is easy to make with a high light efficiency. The field of view (FOV) can be as large as $1^{\circ}$ and be directly put in the front of the focal plane with focal ratio larger than 3 . Such a system for a $60 / 90 / 180$ Schmidt telescope with a $2.5^{\circ}$ objective and a $2048 \times 204815 \mu$ CCD survey system has been designed as an example.

\section{The Fabry-Perot}

If the plate separation is $2.5 \mu$ which is the thickness of one kind of high flatness Dupont Mylar polyester plastic film, the optical path difference is $5 \mu$. We only use a ring-shaped piece of this film to put in between the two FP plates so that the central part of the interferometer is air with refractive index $n=1$. The first light maximum at order $\mathrm{N}=5$ is at wavelength $\lambda=1 \mu$; then $\mathrm{N}=6, \lambda=0.8333 \mu$; $\mathrm{N}=7, \lambda=0.7143 \mu ; \mathrm{N}=8, \lambda=0.6250 \mu ; \mathrm{N}=9, \lambda=0.5555 \mu ; \mathrm{N}=10, \lambda=0.5 \mu ; \mathrm{N}=11$, $\lambda=0.4545 \mu ; \mathrm{N}=12, \lambda=0.4167 \mu ; \mathrm{N}=13, \lambda=0.3846 \mu ; \mathrm{N}=14, \lambda=0.3571 \mu ; \mathrm{N}=15$, 
$\lambda=0.3333 \mu$. So within the thinned back illuminated anti-reflection coated blue CCD's sensitive region we can have 10 light maxima and ten wavelength bands. But for red-sensitive CCD we would only have 7 bands. If the finesse $F=10$, the passband widths are $205,143,104,80,63,51,42,35,30$, and $26 \AA$ respectively. The spectral resolving power $R$ is about 50 to 78 . Normally all these passbands overlap on each other so that we must find a way to separate them.

As we know in FP the finesse $F=\pi \sqrt{(1-r)^{-1}}$, where $\mathbf{r}$ is the reflection coefficient of the inner surface of the two plates. With $m=10$ we need $r=0.73134$ which is not difficult to make. Another parameter is the peak transparency $\tau=\left(1-\alpha(1-r)^{-1}\right)^{2}$ where $\alpha$ is the absorption coefficient of the coating film. If $\alpha$ is less than $2 \%$, the peak transparency will be better than $85 \%$. The FP is an interferometer, so the FOV that can be accepted is $\theta=\sqrt{8 / R} \times d / D$, where $d$ is the diameter of the FP, $D$ is the diameter of the telescope, $R$ is the spectral resolving power we need. With $d=5 \mathrm{~cm}, D=60 \mathrm{~cm}, R \leq 100$, the $F O V$ can be as large as $1^{\circ}$. Because we put it in a convergent beam with focal ratio 3 , this will allow $\mathrm{R} \leq 432$, and the beam angle is less than $10^{\circ}$ which is less than $\sqrt{8 / R}=16.2^{\circ}$.

\section{The choice of the cross disperser}

For $\mathrm{N}=10$, the band width is $46 \AA$ and the pixel size of the Ford $2048 \mathrm{CCD}$ is $15 \mu$, 2 pixels is $30 \mu$, so we need a reciprocal dispersion of $46 / 0.03 \AA / \mathrm{mm}=1533 \AA / \mathrm{mm}$. It is very close with the value for an UBK7 glass made $2.5^{\circ}$ objective prism combined with the 60/90/180 Schmidt telescope. The band width increases with the peak wavelength and the reciprocal dispersion also increases with wavelength. Therefore we can get good pixel and image size matching. The plate scale of the telescope is $114.6 " / \mathrm{mm}, 30 \mu$ equal to 3.4 " which is too large in case of good seeing. It is better to use a telescope with longer focal length or a CCD with smaller pixel size. But still we can get a large FOV ( $0.98^{\circ}$ square).

The total light efficiency $\mathrm{T}=0.96^{4} \times 0.85 \times 0.86 \times 0.5 \times 0.7=0.21$. For a $\mathrm{V}=20.0$ magnitude object, if we integrate 3600 seconds, at $0.5 \mu$ peak, the CCD will collect 1000 electrons which is large enough for a detection and redshift determination.

Acknowledgments. This work was supported by The Natural Sciences Fundation of China. 